\title{
Infinitely many small solutions to an elliptic PDE of variable exponent with a singular nonlinearity
}

\author{
Sekhar Ghosh ${ }^{1}$, Debajyoti Choudhuri ${ }^{1}$, Ratan Kr. Giri ${ }^{2, *}$
}

\begin{abstract}
We prove the existence of infinitely many nonnegative solutions to the following nonlocal elliptic partial differential equation involving singularities

$$
\begin{aligned}
(-\Delta)_{p(\cdot)}^{s} u & =\frac{\lambda}{|u|^{\gamma(x)-1} u}+f(x, u) \text { in } \Omega, \\
u & =0 \text { in } \mathbb{R}^{N} \backslash \Omega
\end{aligned}
$$

where $\Omega \subset \mathbb{R}^{N}, N \geq 2$ is a smooth, bounded domain, $\lambda>0, s \in(0,1), \gamma(x) \in(0,1)$ for all $x \in \bar{\Omega}, N>\operatorname{sp}(x, y)$ for all $(x, y) \in \bar{\Omega} \times \bar{\Omega}$ and $(-\Delta)_{p(\cdot)}^{s}$ is the fractional $p(\cdot)$-Laplacian operator with variable exponent. The nonlinear function $f$ satisfies certain growth conditions. Moreover, we establish a uniform $L^{\infty}(\bar{\Omega})$ estimate of the solution(s) by the Moser iteration technique.

Keywords: Fractional $p(\cdot)$-Laplacian, Variable Order Fractional Sobolev Space, Genus, Symmetric Mountain Pass Theorem, Singularity.

2020 MSC: 35R11, 35J60, 35J75, 46E35.
\end{abstract}

\section{Introduction}

For many a generation of researchers in the field of analysis of elliptic Partial Differential Equations (PDEs) the finding due to Ambrosetti and Rabinowitz [1] still a cornerstone in the study of infinitely many solutions. The authors in [1] considered the Laplacian operator $(-\Delta)$ involving a superlinear data $f$ with the Dirichlet boundary condition in a bounded, Lipschitz domain $\Omega \subset \mathbb{R}^{N}$ and have used the symmetric mountain pass theorem due to Clark [2] to conclude the existence. Mathematically the problem addressed in Ambrosetti and Rabinowitz [1] is as follows.

$$
\begin{aligned}
L u \equiv \Sigma_{i, j=1}^{N} \frac{\partial}{\partial x_{j}}\left(a_{i j}(x) \frac{\partial}{\partial x_{j}} u\right) & =p(x, u) \text { for } x \in \Omega \\
\left.u\right|_{\partial \Omega} & =0 .
\end{aligned}
$$

This led to a chain of works that can be found in [3, 4, 5, 6] and the references therein. Meanwhile, another noteworthy development took place due to Lazer and McKenna [7] in studying elliptic

\footnotetext{
${ }^{*}$ Corresponding author: giri90ratan@gmail.com/giri@campus.technion.ac.il (R.Kr. Giri)

Email addresses: sekharghosh1234@gmail.com (Sekhar Ghosh), dc.iit12@gmail.com (Debajyoti Choudhuri)

${ }^{1}$ Department of Mathematics, National Institute of Technology Rourkela, India

${ }^{2}$ Department of Mathematics, Technion - Israel Institute of Technology, Haifa, Israel
} 
partial differential equations involving a singularity. The problem they considered is

$$
\begin{aligned}
-\Delta u & =p(x) u^{-\gamma} \text { for } x \in \Omega, \\
u & >0 \text { for } x \in \Omega, \\
\left.u\right|_{\partial \Omega} & =0,
\end{aligned}
$$

where $p(x)>0$ in $\bar{\Omega}$ and $\Omega$ is a bounded domain of sufficiently smooth boundary. PDEs involving singularity further gained popularity amongst the researchers with the advent of nonlocal/local operators as well, viz. [8, 9]. Off late the study of existence and multiplicity (finitely many) of solutions have been explored widely for both the local and non local operators. Due to the vast amount of literature it is almost impossible to assimilate all of them here. What we can do is to direct the reader's attention to [10, 11, 12, 13, 14, 15, 16] and the references therein. The work due to [17] is the first of its kind that guarantees the existence of infinitely many positive solutions to a fractional Laplacian problem involving both a singularity and a power nonlinearity with constant exponent. The authors in [17] considered the following problem.

$$
\begin{aligned}
(-\Delta)^{s} u & =\frac{\lambda}{u^{\gamma}}+f(x, u) \text { for } x \in \Omega, \\
u & >0 \text { for } x \in \Omega, \\
u & =0 \text { for } x \in \mathbb{R}^{N} \backslash \Omega,
\end{aligned}
$$

where $\Omega$ is a bounded domain in $\mathbb{R}^{N}$ with a Lipshitz boundary. Recently, PDEs involving operators and nonlinearities of variable orders and exponents respectively took the researchers in a new direction. It is worth mentioning that the literature for the variable order fractional $p(x)$-Laplacian is meager even for the case $p(x)=p, 1<p<\infty$. In Fan [18] the author has introduced with the sub-super solution method for the $p(x)$-Laplacian equations. The equivalence of the local minimizers in the $C^{1}$ topology and the local minimizers in the $W^{1, p(x)}$ topology has been obtained. Further, the author obtained two solutions for an eigenvalue type problem. In [19] the authors have applied the mountain pass theorem to obtain the existence of solutions to the following $p(x)$-Laplacian problem.

$$
\begin{aligned}
-\operatorname{div}\left(a(x)|\nabla u|^{p(x)-2} \nabla u\right)+b(x)|u|^{p(x)-2} u & =f(x, u) \text { in } \Omega, \\
u & =0 \text { on } \partial \Omega,
\end{aligned}
$$

where $1<p^{-} \leq p(x) \leq p^{+}<N, \Omega$ is a bounded domain. Here $f$ could be a superlinear or a sublinear function. The readers may also refer to [20, 21] and the references therein for a study of infinitely many solutiuons. It will be a good read to refer [22] where no Ambrosetti-Rabinowitz type condition has been assumed to obtain multiple solutions to the $p(x)$-Laplacian equations. Some other noteworthy results are due to [23, 24, 25, 26] and the references therein. One may also refer to [27, 28, 29, 30] where in the authors have considered a problem with $p(x)$-Laplacian operator similar to (1.1) with concave-convex nonlinearities.

Off late, the author in [31] studied the new fractional Sobolev space $W^{s, q(x), p(x, y)}(\Omega)$, for $s \in(0,1)$, and the fractional $p(x)$-Laplace operator. Existence of a solution has been guaranteed using a sub-super solution method and a comparison principle involving the fractional $p(x)$-Laplacian. In [32] the authors have developed some qualitative properties of the fractional Sobolev space $W^{s, q(x), p(x, y)}(\Omega)$ for $s \in(0,1)$. Moreover the authos have studied the following problem

$$
\begin{aligned}
L u(x)+|u(x)|^{q(x)-1} u(x) & =\lambda|u(x)|^{r(x)-1} u(x) \text { in } \Omega, \\
u(x) & =0 \text { in } \mathbb{R}^{N} \backslash \Omega,
\end{aligned}
$$


where $\lambda>0,1<r(x)<p^{-}$. One important work which is worth mentioning is due to [33] where the authors have studied the eigenvalue problem involving a fractional $p(x)$-Laplacian. The existence of the eigenvalues and eigenfunctions is based on Ekeland's variational principle. In [34] the authors obtained the multiplicity of solutions for a problem involving a fractional $p(x)$-Laplacian eoparator. Furthermore, the existence of infinitely many solutions to a similar problem can be found in [35]. It is note worthy here to mention that the fractional order derivatives with variable exponents plays an important role to the nonlinear diffusion process [36] when it reacts to the temperature change in particular. Another application is due to Chen et al. [37] to the image restoration. It can be seen from the work due to [38] that the operators with a variable exponent appear in a natural way in continuum mechanics. Further an application of such problems can also bee seen in the elasticity theory [39]. Physicists also appeal to problems involving fractional order Laplacian of variable exponent in their study of fractional quantum mechanics and Levy's path integral [40].

Readers can gain a good amount of information about the fractional Laplacian operator from [41, 42]. The literature survey on problems involving variable order fractional Laplacian and singularity of variable exponent, as far as we know, is almost next to nothing since it is still in the stage of developement. However, motivated from the above mentioned studies and due to the growing interest, we have considered our problem $(\mathrm{P})$ with variable order fractional Laplacian operator involving both singularity and power nonlinearity of variable exponent.

\section{Preliminaries and Main result}

The aim of this paper is to study the following singular elliptic partial differential equation

$$
\begin{aligned}
(-\Delta)_{p(\cdot)}^{s} u & =\frac{\lambda}{|u|^{\gamma(x)-1} u}+f(x, u) \text { in } \Omega, \\
u & =0 \text { in } \mathbb{R}^{N} \backslash \Omega
\end{aligned}
$$

where $\lambda>0, \Omega \subset \mathbb{R}^{N}, N \geq 2$ is a smooth, bounded domain and $s \in(0,1)$ such that $N>\operatorname{sp}(x, y)$ for every $(x, y) \in \bar{\Omega} \times \bar{\Omega}$. The variable exponent function $p(\cdot)$ satisfy the following assumptions.

(i) $p \in C(\bar{\Omega} \times \bar{\Omega}, \mathbb{R})$.

(ii) $p$ is symmetric, i.e. $p(x, y)=p(y, x), \forall(x, y) \in \bar{\Omega} \times \bar{\Omega}$.

(iii) $1<p^{-}=\min _{(x, y) \in \bar{\Omega} \times \bar{\Omega}} p(x, y) \leq p(x, y) \leq p^{+}=\max _{(x, y) \in \bar{\Omega} \times \bar{\Omega}} p(x, y)<\infty$.

The fractional $p(\cdot)$-Laplacian operator, $(-\Delta)_{p(\cdot)}^{s}($ see $[32])$, is defined as

$$
(-\Delta)_{p(\cdot)}^{s} u(x):=P . V . \int_{\mathbb{R}^{n}} \frac{|u(x)-u(y)|^{p(x, y)-2}(u(x)-u(y))}{|x-y|^{N+s p(x, y)}} \mathrm{d} y \text { for all } x \in \mathbb{R}^{N},
$$

where P.V. refers to the Cauchy principal value of an integral. Note that the usual fractional $p$-Laplacian is a special case of this operator with $p(x, y) \equiv$ constant. The singular exponent $\gamma$ satisfies

(i) $\gamma \in C(\bar{\Omega},(0,1))$ and $0<\gamma^{-}=\min _{x \in \bar{\Omega}} \gamma(x) \leq \gamma(x) \leq \gamma^{+}=\max _{x \in \bar{\Omega}} \gamma(x)<1$. 
The function $f$ satisfies the following $p(\cdot)$-sublinear growth conditions.

(i) $f \in C(\bar{\Omega} \times \mathbb{R}, \mathbb{R})$

(ii) $\exists \delta>0$ such that $\forall x \in \Omega$ and $|t| \leq \delta, f(\cdot, t)$ is odd and $\lim _{t \rightarrow 0} \frac{f(x, t)}{t^{\left(p^{-}-1\right)}}=+\infty$ uniformly on $\Omega$

(iii) $\exists \delta^{\prime}>0$ and $\alpha(x) \in\left(1-\gamma(x), p^{-}\right)$with $1-\gamma^{-}<\alpha^{-} \leq \alpha^{+}<p^{-}$such that $\forall x \in \Omega$ and $|t| \leq \delta^{\prime}$, we have $t f(x, t) \leq \alpha(x) F(x, t)$, where $\alpha \in C(\bar{\Omega}, \mathbb{R})$ and $F(x, t)=\int_{0}^{t} f(x, \tau) \mathrm{d} \tau$.

(iv) $1<\alpha^{-}=\min _{x \in \bar{\Omega}} \alpha(x) \leq \alpha(x) \leq \alpha^{+}=\max _{x \in \bar{\Omega}} \alpha(x)<p^{-}$.

It is noteworthy that there are no restrictions on $f$, for $t$ at infinity. The presence of the variable exponent in the problem $\mathrm{P}$ naturally confirms that we will look for solutions in a fractional Sobolev space with variable exponent. We first recall some definitions and notations of variable order Lebesgue spaces due to Rădulescu and Repovs [43]. Let $\Omega \subset \mathbb{R}^{N}$ be a smooth and open domain. Consider the family

$$
C_{+}(\bar{\Omega})=\{q \in C(\bar{\Omega}, \mathbb{R}): q(x)>1, \forall x \in \bar{\Omega}\} .
$$

Define $q^{-}=\inf _{x \in \Omega} q(x)$ and $q^{+}=\sup _{x \in \Omega} q(x)$ for all $q \in C_{+}(\bar{\Omega})$. For all $q \in C_{+}(\bar{\Omega})$, the Lebesgue space of variable order is defined by

$$
L^{q(x)}(\Omega)=\left\{u: u \text { is measurable and } \int_{\Omega}|u(x)|^{q(x)} \mathrm{d} x<\infty\right\}
$$

endowed with the Luxemburg norm

$$
\|u\|_{q(x)}=\inf \left\{\mu>0: \int_{\Omega}\left|\frac{u(x)}{\mu}\right|^{q(x)} \mathrm{d} x \leq 1\right\} .
$$

The space $\left(L^{q(x)}(\Omega),\|\cdot\|\right)$ is a Banach space. Moreover, if $\Omega$ is bounded and the following assumption (2) holds, then the space is separable, reflexive, uniformly convex Banach space ([44, Theorem 1.6, 1.10]).

(i) $1<q^{-}=\min _{x \in \bar{\Omega}} q(x) \leq q(x) \leq q^{+}=\max _{x \in \bar{\Omega}} q(x)<\infty$.

Let $u \in L^{p(x)}(\Omega)$ and $v \in L^{q(x)}(\Omega)$ with $\frac{1}{p(x)}+\frac{1}{q(x)}=1$, then we have the following Hölder type inequality

$$
\left|\int_{\Omega} u v \mathrm{~d} x\right| \leq\left(\frac{1}{p^{-}}+\frac{1}{q^{-}}\right)\|u\|_{p(x)}\|v\|_{q(x)} .
$$

Let us now recall the modular function $\rho_{q(x)}: L^{q(x)}(\Omega) \rightarrow \mathbb{R}$ which plays an important role in the variable order Lebesgue spaces and which is defined by

$$
\rho_{q(x)}(x)=\int_{\Omega}|u(x)|^{q(x)} \mathrm{d} x .
$$

We now state the following Lemma due to [44, Theorem 1.3, 1.4]. 
Lemma 2.1. Suppose, $u, u_{n} \in L^{q(x)}(\Omega)$ for all $n \in \mathbb{N}$. Then

(i) $\|u\|_{q(x)} \lesseqgtr 1$ iff $\rho_{q(x)}(u) \lesseqgtr 1$.

(ii) $\|u\|_{q(x)}<1 \Rightarrow\|u\|_{q(x)}^{q^{+}} \leq \rho_{q(x)}(u) \leq\|u\|_{q(x)}^{q^{-}}$.

(iii) $\|u\|_{q(x)}>1 \Rightarrow\|u\|_{q(x)}^{q^{-}} \leq \rho_{q(x)}(u) \leq\|u\|_{q(x)}^{q^{+}}$.

(iv) $\left\|u_{n}-u\right\|_{q(x)} \rightarrow 0$ iff $\rho_{q(x)}\left(u_{n}-u\right) \rightarrow 0$ iff $u_{n} \rightarrow u$ in measure, in $\Omega$ and $\rho_{q(x)}\left(u_{n}\right) \rightarrow \rho_{q(x)}(u)$.

For the development and properties concerning the Sobolev space $W^{k, p(x)}(\Omega)$ one may refer to [45, 46, 47, 43] and the references therein.

For $0<s<1$, let us now define the variable order fractional Sobolev space as follows. Let

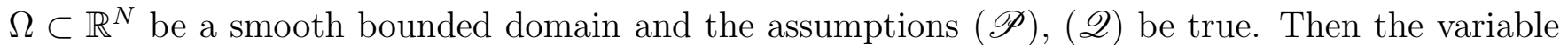
order fractional Sobolev space is defined by

$$
W=W^{s, q(x), p(x, y)}(\Omega)=\left\{u \in L^{q(x)}(\Omega): \iint_{\Omega \times \Omega} \frac{|u(x)-u(y)|^{p(x, y)}}{\mu^{p(x, y)}|x-y|^{N+s p(x, y)}} \mathrm{d} x \mathrm{~d} y<\infty \text { for some } \mu>0\right\} .
$$

The space $\left(W,\|\cdot\|_{W}\right)$ is a Banach space endowed with the natural Gagliardo norm $\|u\|_{W}=\|u\|_{q(x)}+$ $[u]_{s, p(x, y)}$, where $[u]_{s, p(x, y)}$ refers to the Gagliardo semi-norm and is given by

$$
[u]_{s, p(x, y)}=\inf \left\{\mu>0: \iint_{\Omega \times \Omega} \frac{|u(x)-u(y)|^{p(x, y)}}{\mu^{p(x, y)}|x-y|^{N+s p(x, y)}} \mathrm{d} x \mathrm{~d} y<1\right\} .
$$

Moreover, the space $\left(W,\|\cdot\|_{W}\right)$ is separable, reflexive Banach space [31, Lemma 3.1]. Let $W_{0}$ denote the closure of $C_{c}^{\infty}(\Omega)$ in $W$, then $\left(W_{0},\|\cdot\|_{W_{0}}\right)$ is a Banach space equipped with the norm

$$
\|u\|_{W_{0}}=[u]_{s, p(x, y)}=\inf \left\{\mu>0: \iint_{\Omega \times \Omega} \frac{|u(x)-u(y)|^{p(x, y)}}{\mu^{p(x, y)}|x-y|^{N+s p(x, y)}} \mathrm{d} x \mathrm{~d} y<1\right\} .
$$

Similar to the Lemma 2.1, we have the following Lemma for the space $W_{0}$ with the modular function $\rho_{W_{0}}: W_{0} \rightarrow \mathbb{R}$ defined by

$$
\rho_{W_{0}}(u)=\iint_{\Omega \times \Omega} \frac{|u(x)-u(y)|^{p(x, y)}}{|x-y|^{N+s p(x, y)}} \mathrm{d} x \mathrm{~d} y .
$$

Lemma 2.2. Suppose, $u, u_{n} \in W_{0}$ for all $n \in \mathbb{N}$. Then

(i) $\|u\|_{W_{0}} \lesseqgtr 1$ iff $\rho_{W_{0}}(u) \lesseqgtr 1$.

(ii) $\|u\|_{W_{0}}<1 \Rightarrow\|u\|_{W_{0}}^{p^{+}} \leq \rho_{W_{0}}(u) \leq\|u\|_{W_{0}}^{p^{-}}$.

(iii) $\|u\|_{W_{0}}>1 \Rightarrow\|u\|_{W_{0}}^{p^{-}} \leq \rho_{W_{0}}(u) \leq\|u\|_{W_{0}}^{p^{+}}$.

(iv) $\left\|u_{n}-u\right\|_{W_{0}} \rightarrow 0$ iff $\rho_{W_{0}}\left(u_{n}-u\right) \rightarrow 0$ iff $u_{n} \rightarrow u$ in measure in $\Omega$ and $\rho_{W_{0}}\left(u_{n}\right) \rightarrow \rho_{W_{0}}(u)$. 
We now state the following embedding result due to Kaufmann et al. [48, Theorem 1.1].

Theorem 2.3. Let $\Omega \subset \mathbb{R}^{N}$ be a smooth bounded domain and $0<s<1$. Let $q(x)$ and $p(x, y)$

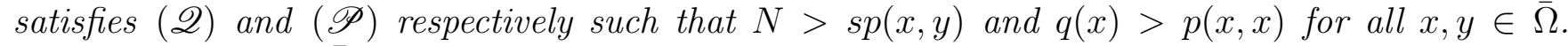
Assume that $r \in C(\bar{\Omega},(1, \infty))$ such that

$$
p_{s}^{*}(x)=\frac{N p(x, x)}{N-\operatorname{sp}(x, x)}>r(x) \geq r^{-}>1, \text { for } x \in \bar{\Omega} .
$$

Then there exists a constant $C=C(N, s, p, q, r, \Omega)$ such that for every $u \in W$ we have

$$
\|u\|_{r(x)} \leq C\|u\|_{W} .
$$

Therefore, the space $W=W^{s, q(x), p(x, y)}(\Omega)$ is continuously embedded into the variable order Lebesgue space $L^{r(x)}(\Omega)$ for all $r \in\left(1, p_{s}^{*}\right)$. Moreover, this embedding is compact. This embedding result holds even for the space $W_{0}$.

Remark 2.4. Theorem 2.3 holds true even for $q(x)=p(x, x)$. See [33, Theorem 2.1] for the proof. Prior to stating our main result, we define a weak solution to the problem (P).

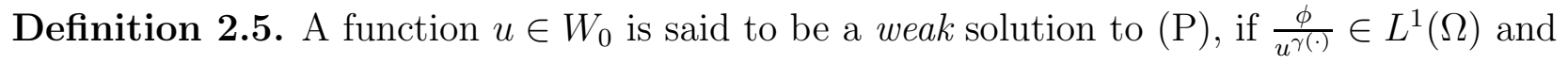

$$
\iint_{\Omega \times \Omega} \frac{|u(x)-u(y)|^{p(x, y)-2}(u(x)-u(y))(\phi(x)-\phi(y))}{|x-y|^{N+s p(x, y)}} \mathrm{d} x \mathrm{~d} y-\int_{\Omega}\left(\frac{\lambda}{|u|^{\gamma(x)-1} u}+f(x, u)\right) \phi \mathrm{d} x=0,
$$

for all $\phi \in W_{0}$.

The associated energy functional $I_{\lambda}: W_{0} \rightarrow(-\infty, \infty]$ for the problem $(\mathbb{P})$ is given by

$$
I_{\lambda}(u):=\iint_{\Omega \times \Omega} \frac{|u(x)-u(y)|^{p(x, y)}}{p(x, y)|x-y|^{N+s p(x, y)}} \mathrm{d} x \mathrm{~d} y-\int_{\Omega} \frac{\lambda}{1-\gamma(x)}|u|^{1-\gamma(x)} \mathrm{d} x-\int_{\Omega} F(x, u) \mathrm{d} x,
$$

where $F(x, t)=\int_{0}^{t} f(x, \tau) \mathrm{d} \tau$. With the above assumptions and definitions we conclude this section by stating the main result of the article.

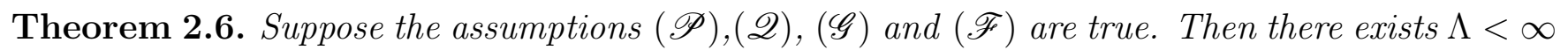
such that for every $\lambda \in(0, \Lambda)$, the problem $(\mathrm{P})$ has infinitely many nonnegative solutions $\left\{u_{n}\right\} \subset$ $W_{0} \cap L^{\infty}(\bar{\Omega})$ with $I_{\lambda}\left(u_{n}\right)<0, I_{\lambda}\left(u_{n}\right) \rightarrow 0^{-}$and $u_{n} \rightarrow 0$ in $W_{0}$.

\section{Existence of infinitely many solutions}

This section is entirely devoted to obtain infinitely many solutions with the help of symmetric Mountain Pass Theorem due to Kajikiya [3, Theorem 1(i)]. The main difficulty to apply the symmetric Mountain Pass Theorem is that the functional $I_{\lambda}$ fails to be $C^{1}(\Omega)$ due the presence of the singular term. Therefore, we first modify the problem $(\mathbb{P})$ in the neighbourhood of 0 by employing a cut-off technique developed in [2], which can also be found in [3]. Suppose the assumptions (盾) holds true. Choose $0<l \leq \frac{1}{2} \min \left\{\delta, \delta^{\prime}\right\}$, where $\delta, \delta^{\prime}>0$ are as in $(\mathscr{F}$. Let us define a bounded $C^{1}$ function $\xi: \mathbb{R} \rightarrow \mathbb{R}^{+}$with $0 \leq \xi(t) \leq 1$ such that $\xi(t)=1$, if $|t| \leq l, \xi(t)=0$ if $|t| \geq 2 l$, $\xi$ is 
decreasing in $[l, 2 l]$ and increasing in $[-2 l,-l]$ and define $\bar{f}(x, u)=f(x, u) \xi(u)$. Now consider the following cut-off problem

$$
\begin{aligned}
(-\Delta)_{p(\cdot)}^{s} u & =\frac{\lambda}{|u|^{\gamma(x)-1} u}+\bar{f}(x, u) \text { in } \Omega \\
u & =0 \text { in } \mathbb{R}^{N} \backslash \Omega .
\end{aligned}
$$

Then we say that a function $u \in W_{0}$ is a weak solution of (3.1), if $\frac{\phi}{u^{\gamma(\cdot)}} \in L^{1}(\Omega)$ and

$$
\iint_{\Omega \times \Omega} \frac{|u(x)-u(y)|^{p(x, y)-2}(u(x)-u(y))(\phi(x)-\phi(y))}{|x-y|^{N+s p(x, y)}} \mathrm{d} x \mathrm{~d} y-\int_{\Omega}\left(\frac{\lambda}{|u|^{\gamma(x)-1} u}+\bar{f}(x, u)\right) \phi \mathrm{d} x=0
$$

for all $\phi \in W_{0}$. The associated energy functional $\bar{I}_{\lambda}: W_{0} \rightarrow(-\infty, \infty]$ corresponding to the problem (3.1) is given by

$$
\bar{I}_{\lambda}(u)=\iint_{\Omega \times \Omega} \frac{|u(x)-u(y)|^{p(x, y)}}{p(x, y)|x-y|^{N+s p(x, y)}} \mathrm{d} x \mathrm{~d} y-\int_{\Omega} \frac{\lambda}{1-\gamma(x)}|u|^{1-\gamma(x)} \mathrm{d} x-\int_{\Omega} \bar{F}(x, u) \mathrm{d} x,
$$

where $\bar{F}(x, t)=\int_{0}^{t} \bar{f}(x, \tau) d \tau$. It is easy to see that if $u$ is a solution to the problem (3.1) with $\|u\|_{\infty} \leq l$ then $u$ is also a solution to the problem $(\mathrm{P})$. Hence we will obtain infinitely many solutions to the problem (3.1) such that $\|u\|_{\infty} \leq l$. Prior to proving the existence results, let us recall the statement of symmetric Mountain Pass Theorem due to Kajikiya [3] followed by the definition of genus of a set.

Definition 3.1 (Genus [3]). Let $X$ be a Banach space and $A \subset X$. A set $A$ is said to be symmetric if $u \in A$ implies $-u \in A$. Let $A$ be a close, symmetric subset of $X$ such that $0 \notin A$. We define a genus $\gamma(A)$ of $A$ by the smallest integer $k$ such that there exists an odd continuous mapping from $A$ to $\mathbb{R}^{k} \backslash\{0\}$. We define $\gamma(A)=\infty$, if no such $k$ exists.

Theorem 3.2 (Symmetric Mountain Pass Theorem [3]). Let $X$ be an infinite dimensional Banach space and $I \in C^{1}(X, \mathbb{R})$ satisfies the following

(i) I is even, bounded below, $I(0)=0$ and I satifies the $(P S)_{c}$ condition.

(ii) For each $n \in \mathbb{N}$, there exists an $A_{n} \in \Gamma_{n}$ such that $\sup _{u \in A_{n}} I(u)<0$.

Then for each $n \in \mathbb{N}, c_{n}=\inf _{A \in \Gamma_{n}} \sup _{u \in A} I(u)<0$ is a critical value of $I$.

We first state and prove the following Lemma which gives a finite range of $\lambda$.

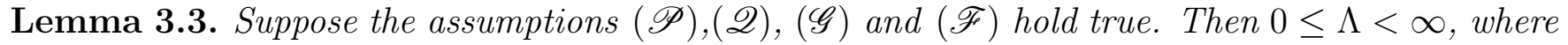
$\Lambda=\inf \{\lambda>0$ : The problem $(\mathbb{P})$ has no solution $\}$.

Proof. It can be easily seen that $\Lambda \geq 0$. What we are left to check is whether $\Lambda<\infty$ or not. This will be proved by contradiction. Let $\Lambda=\infty$. Then it can be assumed that there exists $\lambda_{n} \rightarrow \infty$ such that $u_{n}$ is a solution to the problem (P). On choosing $\lambda_{*}>0$ such that

$$
\lambda t^{-\gamma(x)}+t^{\alpha(x)}>\left(\lambda_{1}+\epsilon\right) t^{p(x)-1}, \forall t>0, \epsilon \in(0,1), \lambda>\lambda_{*} .
$$


Corresponding to each choice of $\lambda_{n}>\lambda_{*}$ we have $u_{n}$, a solution of the problem (IP). Note that $\bar{u}=u_{\lambda}$ is a supersolution to the following problem.

$$
\begin{aligned}
\left(-\Delta_{p}(x)\right)^{s} u & =\left(\lambda_{1}+\epsilon\right)|u|^{p(x)-2} u \text { in } \Omega, \\
u & =0 \text { in } \mathbb{R}^{N} \backslash \Omega .
\end{aligned}
$$

Further, choose $r>0$ small enough such that $\underline{u}=r \phi_{1}$ is a subsolution to (3.4). Since, $u_{\lambda}, \phi_{1} \in$ $L^{\infty}(\Omega)$ then there exists $r>0$ sufficiently small such that $\underline{u} \leq \bar{u}$. Consider the monotone iteration

$$
\begin{aligned}
u_{0} & =r \phi_{1} \\
\left(-\Delta_{p}(x)\right)^{s} u_{n} & =\left(\lambda_{1}+\epsilon\right) u_{n-1}^{p(x)-1} \text { and } u_{n}>0 \text { in } \Omega
\end{aligned}
$$

Therefore, by using the weak comparison principle we have $r \phi_{1} \leq u_{1}(x) \leq u_{2}(x) \leq \cdots \leq u_{n}(x) \leq$ $\cdots \leq u_{\lambda}(x), \forall x \in \Omega$. Therefore the sequence $\left\{u_{n}\right\}$ is bounded in $X_{0}$ and hence $\left\{u_{n}\right\}$ has a weakly convergent subsequence, still denoted by $\left\{u_{n}\right\}$, converging to $u_{0}$, which is a solution to the problem (3.4). Since, $\epsilon>0$ is arbitrary and $\lambda_{1}$ is simple and isolated, hence a contradiction is arrived at. Thus one can conclude that $\Lambda<\infty$.

The next two consecutive Lemmas guarantees the validity of the hypothesis $(i)$ and $(i i)$ of the Theorem 3.2 for the functional $\bar{I}_{\lambda}$.

Lemma 3.4. The functional $\bar{I}_{\lambda}$ is bounded below, even, $\bar{I}_{\lambda}(0)=0$ and satisfies $(P S)_{c}$ condition.

Proof. Clearly, $\bar{I}_{\lambda}(0)=0$ and even. We now prove that the functional is bounded from below. Without loss of generality let us assume $\|u\|_{W_{0}}>1$. Then by virtue of the Hölder's inequality (2.1) together with the compact embedding, Theorem 2.3 and by using the definition of $\xi$, we get

$$
\begin{aligned}
\bar{I}_{\lambda}(u) & \geq \frac{1}{p^{+}} \iint_{\Omega \times \Omega} \frac{|u(x)-u(y)|^{p(x, y)}}{|x-y|^{N+s p(x, y)}} \mathrm{d} x \mathrm{~d} y-\frac{\lambda}{1-\gamma^{+}} \int_{\Omega}|u|^{1-\gamma(x)} \mathrm{d} x-C \\
& \geq \frac{1}{p^{+}}\|u\|_{W_{0}}^{p^{-}}-\frac{C^{\prime} \lambda}{1-\gamma^{+}}\|u\|_{W_{0}}^{1-\gamma^{+}}-C,
\end{aligned}
$$

where $C, C^{\prime}$ are positive constants. This guarantees that $\bar{I}_{\lambda}$ is coercive as well as bounded from below. Now suppose $\left\{u_{n}\right\} \subset W_{0}$ is a $(P S)_{c}$ sequence for $\bar{I}_{\lambda}$. Therefore, by using the coercivity of $\bar{I}_{\lambda}$, we have that the sequence $\left\{u_{n}\right\}$ is bounded and hence upto a subsequence $u_{n} \rightarrow u$ in $W_{0}$. Hence, for every $\phi \in W_{0}$, we get

$$
\begin{aligned}
\iint_{\Omega \times \Omega} & \frac{\left|u_{n}(x)-u_{n}(y)\right|^{p(x, y)-2}\left(u_{n}(x)-u_{n}(y)\right)(\phi(x)-\phi(y))}{|x-y|^{N+s p(x, y)}} \mathrm{d} x \mathrm{~d} y \\
& \longrightarrow \iint_{\Omega \times \Omega} \frac{|u(x)-u(y)|^{p(x, y)-2}(u(x)-u(y))(\phi(x)-\phi(y))}{|x-y|^{N+s p(x, y)}} \mathrm{d} x \mathrm{~d} y .
\end{aligned}
$$

Moreover, by the Theorem 2.3, we have as $n \rightarrow \infty$

$$
u_{n} \longrightarrow u \text { in } L^{r(x)}(\Omega), \forall 1<r(x)<p_{s}^{*}(x) \text { and } u_{n}(x) \longrightarrow u(x) \text { a.e. } \Omega \text {. }
$$


Claim: Under the assumption $(\underline{\mathscr{F}})$, we have

$$
\int_{\Omega} \bar{f}\left(x, u_{n}\right) u \mathrm{~d} x \rightarrow \int_{\Omega} \bar{f}(x, u) u \mathrm{~d} x \text { and } \int_{\Omega} \bar{f}\left(x, u_{n}\right) u_{n} \mathrm{~d} x \rightarrow \int_{\Omega} \bar{f}(x, u) u \mathrm{~d} x .
$$

By using (3.7) and Lemma 5.2, there exists a $g \in L^{r(x)}(\Omega)$ such that

$$
\left|u_{n}(x)\right| \leq g(x) \text { a.e. in } \Omega, \forall n \in \mathbb{N} \text {. }
$$

Therefore, from (3.7), (3.9) and by using Lebesgue dominated convergence theorem and a BrezisLieb type Lemma [49], our claim is established.

Now on using the Hölder's inequality (2.1) with $r_{1}(x)$ and $r_{1}^{\prime}(x)$ such that $r_{1}(x)(1-\gamma(x))>1$ and then on passing the limit $n \rightarrow \infty$, we have

$$
\begin{aligned}
\int_{\Omega}\left|u_{n}\right|^{1-\gamma(x)} \mathrm{d} x & \leq \int_{\Omega}|u|^{1-\gamma(x)} \mathrm{d} x+\int_{\Omega}\left|u_{n}-u\right|^{1-\gamma(x)} \mathrm{d} x \\
& \leq \int_{\Omega}|u|^{1-\gamma(x)} \mathrm{d} x+C\left\|u_{n}-u\right\|_{L^{r_{1}(x)}(\Omega)}^{1-\gamma^{+}} \\
& =\int_{\Omega}|u|^{1-\gamma(x)} \mathrm{d} x+o(1)
\end{aligned}
$$

On proceeding in similar way, we obtain

$$
\int_{\Omega}|u|^{1-\gamma} d x \leq \int_{\Omega}\left|u_{n}\right|^{1-\gamma} \mathrm{d} x+o(1)
$$

Therefore, from (3.10) and (3.11) it follows that

$$
\int_{\Omega} u_{n}^{1-\gamma} d x=\int_{\Omega} u^{1-\gamma} \mathrm{d} x+o(1)
$$

Now by using the fact that $\left\langle\bar{I}_{\lambda}^{\prime}\left(u_{n}\right), u_{n}\right\rangle \rightarrow 0$ as $n \rightarrow \infty$, we get

$$
\iint_{\Omega \times \Omega} \frac{\left|u_{n}(x)-u_{n}(y)\right|^{p(x, y)}}{|x-y|^{N+s p(x, y)}} \mathrm{d} x \mathrm{~d} y-\lambda \int_{\Omega}\left|u_{n}\right|^{1-\gamma(x)} \mathrm{d} x-\int_{\Omega} \bar{f}\left(x, u_{n}\right) u_{n} \mathrm{~d} x \rightarrow 0
$$

Hence the estimates (3.8), (3.12) and (3.13) gives

$$
\iint_{\Omega \times \Omega} \frac{|u(x)-u(y)|^{p(x, y)}}{|x-y|^{N+s p(x, y)}} \mathrm{d} x \mathrm{~d} y \rightarrow \lambda \int_{\Omega}|u|^{1-\gamma(x)} \mathrm{d} x+\int_{\Omega} \bar{f}(x, u) u \mathrm{~d} x
$$

Again, we have $\left\langle\bar{I}_{\lambda}^{\prime}\left(u_{n}\right), u\right\rangle \longrightarrow 0$ as $n \rightarrow \infty$ and

$$
\begin{gathered}
\left\langle\bar{I}_{\lambda}^{\prime}\left(u_{n}\right), u\right\rangle=\iint_{\Omega \times \Omega} \frac{\left|u_{n}(x)-u_{n}(y)\right|^{p(x, y)-2}\left(u_{n}(x)-u_{n}(y)\right)(u(x)-u(y))}{|x-y|^{N+s p(x, y)}} \mathrm{d} x \mathrm{~d} y \\
-\lambda \int_{\Omega} \frac{u}{\left|u_{n}\right|^{\gamma(x)-1} u_{n}} d x-\int_{\Omega} \bar{f}\left(x, u_{n}\right) u d x
\end{gathered}
$$

Therefore, on choosing $\phi=u$ as the test function in (3.6) and then by using (3.12)-(3.14), we obtain

$$
\iint_{\Omega \times \Omega} \frac{|u(x)-u(y)|^{p(x, y)}}{|x-y|^{N+s p(x, y)}} \mathrm{d} x \mathrm{~d} y=\lambda \int_{\Omega}|u|^{1-\gamma(x)} \mathrm{d} x+\int_{\Omega} \bar{f}(x, u) u \mathrm{~d} x
$$

Hence, $\left\|u_{n}\right\|_{W_{0}} \rightarrow\|u\|_{W_{0}}$ which guarantees that the functional $\bar{I}_{\lambda}$ satisfies $(P S)_{c}$ condition. 
Define $\Gamma_{n}=\left\{A_{n} \subset W_{0}: A_{n}\right.$ is closed, symmetric and $0 \notin A_{n}$ such that the genus $\left.\gamma\left(A_{n}\right) \geq n\right\}$.

Lemma 3.5. For any $n \in \mathbb{N}$, there exists a closed, symmetric subset $A_{n} \subset W_{0}$ with $0 \notin A_{n}$ such that $\gamma\left(A_{n}\right) \geq n$ and $\sup _{u \in A_{n}} \bar{I}_{\lambda}(u)<0$.

Proof. We first construct a finite dimensional subspace of $W_{0}$. Observe that by using the definition of $\xi$, there exists $R>0$ such that $\bar{f}(x, t) \leq R$ and $\bar{F}(x, t) \leq R$ for all $(x, t) \in \Omega \times \mathbb{R}$. Now since $\alpha^{+}<p^{-}$, then by using the definition of $\bar{f}$, we get

$$
\bar{F}(x, u)=\xi(u) f(x, u) \geq \frac{C|u|^{\alpha^{+}}}{\alpha^{+}} \forall x \in \Omega \text { and }|u| \leq l,
$$

for some $C>0$ and $0<l \leq 1$. Now for a fix $k \in \mathbb{N}$, choose, $\left\{\phi_{1}, \phi_{2}, \cdots, \phi_{k}\right\} \subset C_{0}^{\infty}(\Omega)$ such that $\phi_{i} \neq 0, \operatorname{supp}\left(\phi_{i}\right) \subset \Omega$ for all $i=1,2, \cdots, k$ and $\operatorname{supp}\left(\phi_{i}\right) \cap \operatorname{supp}\left(\phi_{j}\right)=\emptyset$ for $i \neq j$. Define, $W_{k}=\operatorname{span}\left\{\phi_{1}, \phi_{2}, \cdots, \phi_{k}\right\}$. Clearly $W_{k}$ is a finite subspace of $W_{0}$. Now, since all norms on a finite dimensional subspace are equivalent then there exist $c_{k}, d_{k}>0$ such that

$$
\|u\|_{W_{0}} \geq c_{k}\|u\|_{\infty} \text { and }\|u\|_{\alpha^{+}} \leq d_{k}\|u\|_{W_{0}} .
$$

Finally, on choosing $\rho_{k}=\left\{\frac{l}{2}, \frac{l c_{k}}{2},\left(\frac{C l p^{-} d_{k}^{+}}{2 \alpha^{+}}\right)^{\frac{1}{p^{-}-\alpha^{+}}}\right\}$and by using Lemma 2.2, (3.17), (3.18), we get

$$
\begin{aligned}
\bar{I}_{\lambda}(u) & \leq \frac{1}{p^{-}} \iint_{\Omega \times \Omega} \frac{|u(x)-u(y)|^{p(x, y)}}{|x-y|^{N+s p(x, y)}} \mathrm{d} x \mathrm{~d} y-\frac{\lambda}{1-\gamma^{-}} \int_{\Omega}|u|^{1-\gamma(x)} \mathrm{d} x-\int_{\Omega} \bar{F}(x, u) \mathrm{d} x \\
& \leq \frac{1}{p^{-}}\|u\|_{W_{0}}^{p^{-}}-\frac{\lambda}{1-\gamma^{-}} \int_{\Omega}|u|^{1-\gamma(x)} \mathrm{d} x-\frac{C}{\alpha^{+}} \int_{\Omega}|u|^{\alpha(x)} \mathrm{d} x \\
& \leq\left[\frac{1}{p^{-}}\|u\|_{W_{0}}^{p^{-}}-\frac{C d_{k}^{\alpha^{+}}}{\alpha^{+}}\|u\|_{W_{0}}^{\alpha^{+}} \mathrm{d} x\right]-\frac{\lambda}{1-\gamma^{-}} \int_{\Omega}|u|^{1-\gamma(x)} \mathrm{d} x \\
& <0
\end{aligned}
$$

for all $u \in W_{k} \cap S_{\rho_{k}}$, where $S_{\rho}=\left\{u \in W_{k}:\|u\|_{W_{0}}=\rho\right\}$. Set, $A_{n}:=\left\{u \in W_{n}:\|u\|_{W_{0}}=\rho_{n}\right\}$. Then $\Gamma_{n} \neq \phi$. Moreover, $A_{n}$ is symmetric, closed such that $\gamma\left(A_{n}\right) \geq n$ and $\sup _{u \in A_{n}} \bar{I}_{\lambda}(u)<0$. This completes the proof.

Proof of Theorem 2.6. We can see that Lemma 3.4 and Lemma 3.5 satisfies the hypothesis $(i)$ and $(i i)$ of Theorem [3.2. Therefore, we conclude that the functional $\bar{I}_{\lambda}$ has sequence of critical points $\left\{u_{n}\right\}$ such that $\bar{I}_{\lambda}\left(u_{n}\right)<0$ and $\bar{I}_{\lambda}\left(u_{n}\right) \rightarrow 0^{-}$. We first prove the nonnegativity of solutions to the problem $(\mathrm{P})$. Consider $\Omega=\Omega^{+} \cup \Omega^{-}$, where $\Omega^{+}=\left\{x \in W_{0}: u_{n}(x) \geq 0\right\}$ and $\Omega^{-}=$ $\left\{x \in W_{0}: u_{n}(x)<0\right\}$. Also, let us define $u_{n}(x)=u_{n}^{+}-u_{n}^{-}$, where $u_{n}^{+}(x)=\max \left\{u_{n}(x), 0\right\}$ and $u_{n}^{-}(x)=\max \left\{-u_{n}(x), 0\right\}$. We will proceed by the method of contradiction. Suppose, $u_{n}<0$ a.e. in $\Omega$. On choosing, $\phi=u_{n}^{-}$as the test function in (2.2) and using $(a-b)\left(a^{-}-b^{-}\right) \leq-\left(a^{-}-b^{-}\right)^{2}$, 
we have

$$
\begin{aligned}
& \int_{\Omega}\left(\lambda \frac{u_{n}^{-}}{\left|u_{n}\right|^{\gamma(x)-1} u_{n}}+f\left(x, u_{n}\right) u_{n}^{-}\right) \mathrm{d} x=\iint_{\Omega \times \Omega} \frac{\left|u_{n}(x)-u_{n}(y)\right|^{p(x, y)-2}\left(u_{n}(x)-u_{n}(y)\right)\left(u_{n}^{-}(x)-u_{n}^{-}(y)\right)}{|x-y|^{N+2 s}} \mathrm{~d} x \mathrm{~d} y \\
& \Rightarrow \lambda \int_{\Omega} \frac{\operatorname{sign}\left(u_{n}\right) u_{n}^{-}}{\left|u_{n}\right|^{\gamma(x)}} \mathrm{d} x \leq-\iint_{\Omega \times \Omega} \frac{\left|u_{n}(x)-u_{n}(y)\right|^{p(x, y)-2}\left(u_{n}^{-}(x)-u_{n}^{-}(y)\right)^{2}}{|x-y|^{N+2 s}} \mathrm{~d} x \mathrm{~d} y \\
& \Rightarrow \lambda \int_{\Omega^{-}}\left|u_{n}^{-}\right|^{1-\gamma(x)} d x \leq-\rho_{W_{0}}\left(u_{n}^{-}\right)<0 .
\end{aligned}
$$

Therefore, by using Lemma 2.2, we can conclude that $\left|\Omega^{-}\right|=0$. This contradicts the assumption $u_{n}<0$ a.e. in $\Omega$ and hence the solutions to $(\mathrm{P})$ are nonnegative. Now observe that $o_{n}(1)=$ $\frac{1}{\alpha^{+}}\left\langle\bar{I}_{\lambda}^{\prime}\left(u_{n}\right), u_{n}\right\rangle-\bar{I}_{\lambda}\left(u_{n}\right)$. Moreover, from the definition of $\bar{I}_{\lambda}$, we have

$$
\begin{aligned}
\frac{1}{\alpha^{+}}\left\langle\tilde{I}^{\prime}\left(u_{n}\right), u_{n}\right\rangle-\tilde{I}\left(u_{n}\right)= & \frac{1}{\alpha^{+}}\left[\iint_{\Omega \times \Omega} \frac{\left|u_{n}(x)-u_{n}(y)\right|^{p(x, y)}}{|x-y|^{N+s p(x, y)}} \mathrm{d} x \mathrm{~d} y-\int_{\Omega}\left(\lambda\left|u_{n}\right|^{1-\gamma(x)}+\bar{f}\left(x, u_{n}\right) u_{n}\right) \mathrm{d} x\right] \\
& -\left[\iint_{\Omega \times \Omega} \frac{\left|u_{n}(x)-u_{n}(y)\right|^{p(x, y)}}{p(x, y)|x-y|^{N+s p(x, y)}} \mathrm{d} x \mathrm{~d} y-\int_{\Omega}\left(\frac{\lambda\left|u_{n}\right|^{1-\gamma(x)}}{1-\gamma(x)}+\bar{F}\left(x, u_{n}\right)\right) \mathrm{d} x\right] \\
\geq & \left(\frac{1}{\alpha^{+}}-\frac{1}{p^{-}}\right) \iint_{\Omega \times \Omega} \frac{\left|u_{n}(x)-u_{n}(y)\right|^{p(x, y)}}{|x-y|^{N+s p(x, y)}} \mathrm{d} x \mathrm{~d} y \\
& -\lambda\left(\frac{1}{\alpha^{+}}-\frac{1}{1-\gamma^{-}}\right) \int_{\Omega}\left|u_{n}\right|^{1-\gamma(x)} \mathrm{d} x+\frac{1}{\alpha^{+}} \int_{\Omega}\left(\alpha^{+} \bar{F}\left(x, u_{n}\right)-\bar{f}\left(x, u_{n}\right)\right) \mathrm{d} x \\
\geq & \left(\frac{1}{\alpha^{+}}-\frac{1}{p^{-}}\right) \rho_{W_{0}}\left(u_{n}\right)+\lambda\left(\frac{1}{1-\gamma^{-}}-\frac{1}{\alpha^{+}}\right) \int_{\Omega}\left|u_{n}\right|^{1-\gamma(x)} \mathrm{d} x \\
\geq & \left(\frac{1}{\alpha^{+}}-\frac{1}{p^{-}}\right) \rho_{W_{0}}\left(u_{n}\right) .
\end{aligned}
$$

Therefore, $o_{n}(1)=\frac{1}{\alpha^{+}}\left\langle\bar{I}_{\lambda}^{\prime}\left(u_{n}\right), u_{n}\right\rangle-\bar{I}_{\lambda}\left(u_{n}\right) \geq\left(\frac{1}{\alpha^{+}}-\frac{1}{p^{-}}\right) \rho_{W_{0}}\left(u_{n}\right)$. Hence, we get $\rho_{W_{0}}\left(u_{n}\right) \rightarrow 0$ and this implies that $u_{n} \rightarrow 0$ in $W_{0}$. Thus, we can conclude that the problem (3.1) has infinitely many solutions. Finally, thanks to the Moser iteration, from Lemma 4.6, we can obtain $\left\|u_{n}\right\|_{L^{\infty}(\Omega)} \leq l$ as $n \rightarrow \infty$. Therefore, the problem (P) has infinitely many nonnegative weak solutions.

\section{Boundedness of solutions to $(\mathrm{P})$.}

In this section we obtain a uniform bound of weak solutions to the problem (3.1) by using Moser iteration method followed by some auxilliary Lemmas. Let us begin with the following Lemma.

Lemma 4.1. For every $\beta(x)>0$ and $p(x) \geq 1$ we have

$$
\left(\frac{1}{\beta(x)}\right)^{\frac{1}{p(x)}}\left(\frac{p(x)+\beta(x)-1}{p(x)}\right) \geq 1 .
$$

Proof. Observe that for $p(x)=1$, the result is obvious. Therefore, we may assume $p(x)>1$. Now for $p(x)>1$ the function $t \mapsto t^{p(x)}$ is convex. Hence,

$$
\beta(x)-1 \geq p(x)\left(\beta(x)^{\frac{1}{p(x)}}-1\right) .
$$


Now on adding $p(x)$ to both sides we obtain

$$
p(x)+\beta(x)-1 \geq p(x) \beta(x)^{\frac{1}{p(x)}} .
$$

Hence, we get

$$
\left(\frac{1}{\beta(x)}\right)^{\frac{1}{p(x)}}\left(\frac{p(x)+\beta(x)-1}{p(x)}\right) \geq 1 .
$$

We now consider the monotone increasing function $J_{p(x)}(t):=|t|^{p(x)-2} t$ for every $1<p(x)<\infty$.

Lemma 4.2. Assume $1<p(x)<\infty$ and $f: \mathbb{R} \rightarrow \mathbb{R}$ to be a $C^{1}$ convex function. Then for any $\tau \geq 0$

$J_{p(x)}(a-b)\left[A J_{p(x), \tau}\left(f^{\prime}(a)\right)-B J_{p(x), \tau}\left(f^{\prime}(b)\right)\right] \geq\left(\tau(a-b)^{2}+(f(a)-f(b))^{2}\right)^{\frac{p(x)-2}{2}}(f(a)-f(b))(A-B)$,

for every $a, b \in \mathbb{R}$ and every $A, B \geq 0$, where $J_{p(x), \tau}(t)=\left(\tau+|t|^{2}\right)^{\frac{p-2}{2}} t, t \in \mathbb{R}$.

Proof. The result is trivial if $a=b$. Therefore, let us assume $a \neq b$. Since the function $f$ is $C^{1}$ and convex then

$$
f(a)-f(b) \leq f^{\prime}(a)(a-b) \text { and } f(a)-f(b) \geq f^{\prime}(b)(a-b)
$$

Now from the left hand side of (4.1), we get

$$
\begin{aligned}
J_{p(x)}(a-b) & {\left[A J_{p(x), \tau}\left(f^{\prime}(a)\right)-B J_{p(x), \tau}\left(f^{\prime}(b)\right)\right] } \\
& =\left(\tau(a-b)^{2}+\left(f^{\prime}(a)(a-b)\right)^{2}\right)^{\frac{p(x)-2}{2}} f^{\prime}(a)(a-b) A \\
& -\left(\tau(a-b)^{2}+\left(f^{\prime}(b)(a-b)\right)^{2}\right)^{\frac{p(x)-2}{2}} f^{\prime}(b)(a-b) B,
\end{aligned}
$$

Observe that the function $F(t)=\frac{1}{p(x)}\left(\tau(a-b)^{2}+t^{2}\right)^{\frac{p(x)}{2}}$ is convex and its derivative is given by $F^{\prime}(t)=\left(\tau(a-b)^{2}+t^{2}\right)^{\frac{p(x)-2}{2}} t$. Then by simplifying (4.2) and by the monotonicity of $F^{\prime}$, we can obtain the desired inequality (4.1).

Remark 4.3. For $\tau=0$, the estimate of the above Lemma becomes

$$
J_{p(x)}(a-b)\left[A J_{p(x)}\left(f^{\prime}(a)\right)-B J_{p(x)}\left(f^{\prime}(b)\right)\right] \geq(f(a)-f(b))^{p(x)-2}(f(a)-f(b))(A-B),
$$

for every $a, b \in \mathbb{R}$ and every $A, B \geq 0$

Lemma 4.4. Assume $1<p(x)<\infty$ and $g: \mathbb{R} \rightarrow \mathbb{R}$ to be an increasing function. Define

$$
G(t)=\int_{0}^{t} g^{\prime}(\tau)^{\frac{1}{p(x)}} \mathrm{d} \tau, t \in \mathbb{R}
$$

then we have

$$
J_{p(x)}(a-b)(g(a)-g(b)) \geq|G(a)-G(b)|^{p(x)} .
$$


Proof. Suppose $a>b$ without the loss of generality. Then we have

$$
\begin{aligned}
J_{p(x)}(a-b)(g(a)-g(b)) & =(a-b)^{p(x)-1} \int_{b}^{a} g^{\prime}(\tau) \mathrm{d} \tau \\
& =(a-b)^{p(x)-1} \int_{b}^{a} G^{\prime}(\tau)^{p(x)} \mathrm{d} \tau \\
& \geq\left(\int_{b}^{a} G^{\prime}(\tau) \mathrm{d} \tau\right)^{p(x)} \quad \text { (by using Jensen inequality) } \\
& =|G(a)-G(b)|^{p(x)}
\end{aligned}
$$

Lemma 4.5. Suppose $\beta(x) \geq 1$, then for every $a, b \geq 0$ we have

$$
|a-b|^{p(x)}\left(a^{\beta(x)-1}+b^{\beta(x)-1}\right) \leq(\max \{1,(3-\beta(x))\})|a-b|^{p(x)-2}(a-b)\left(a^{\beta(x)}-b^{\beta(x)}\right) .
$$

Proof. Observe that the estimate (4.5) is true for $a=b$. Therefore, one can assume $a>b$. Then the estimate (4.5) reduces to

$$
(1-t)^{p(x)}\left(1+t^{\beta(x)-1}\right) \leq C(1-t)^{p(x)-1}\left(1-t^{\beta(x)}\right) \text { for } 0 \leq t<1,
$$

which implies that

$$
(1-t)\left(1+t^{\beta(x)-1}\right) \leq C(1-t)\left(1-t^{\beta(x)}\right) \text { for } 0 \leq t<1
$$

Note that $(1-t)\left(1+t^{\beta(x)-1}\right)=\left(1-t^{\beta(x)}\right)+t^{\beta(x)-1}-t$. Since $0 \leq t<1$, then for every $\beta(x) \geq 2$ we have $t^{\beta(x)-1}-t \leq 0$. On the other hand for $1<\beta(x)<2$, the function $\tau \mapsto \tau^{\beta(x)-1}$ is concave. Therefore, on using the concavity we get

$$
t^{\beta(x)-1}-t=\left(t^{\beta(x)-1}-1\right)-(t-1) \leq(\beta(x)-1)(t-1)-(t-1) \leq(2-\beta(x))\left(1-t^{\beta(x)}\right) .
$$

The estimate (4.5) holds trivially for $\beta(x)=1$. Hence we can conclude the proof for every $\beta(x) \geq$ 1.

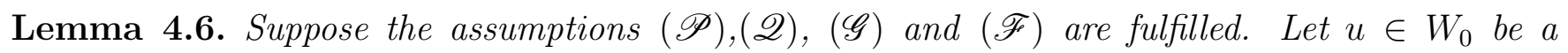
solution to the problem (3.1). Then $u \in L^{\infty}(\bar{\Omega})$.

Proof. Let us first define the smooth function $g_{\epsilon}(t)=\left(\epsilon^{2}+t^{2}\right)^{\frac{1}{2}}$ for every $\epsilon>0$. Note that the function $g_{\epsilon}$ is convex and Lipschitz. For each $0<\psi \in C_{c}^{\infty}(\Omega)$, we choose $\phi=\psi\left|g_{\epsilon}^{\prime}(u)\right|^{p(x, y)-2} g_{\epsilon}^{\prime}(u)$ as the test function in (3.2). By choosing $a=u(x), b=u(y), A=\psi(x)$ and $B=\psi(y)$ in Lemma 4.2 we have

$$
\begin{gathered}
\iint_{\Omega \times \Omega} \frac{\left|g_{\epsilon}(u(x))-g_{\epsilon}(u(y))\right|^{p(x, y)-2}\left(g_{\epsilon}(u(x))-g_{\epsilon}(u(y))\right)(\psi(x)-\psi(y))}{|x-y|^{N+s p(x, y)}} \mathrm{d} x \mathrm{~d} y \\
\quad \leq \int_{\Omega}\left(\left|\frac{\lambda}{|u|^{\gamma(x)-1} u}+\bar{f}(x, u)\right|\right)\left|g_{\epsilon}(u)\right|^{p(x)-1} \psi \mathrm{d} x
\end{gathered}
$$


for all $0<\psi \in C_{c}^{\infty}(\Omega)$. Moreover, $g_{\epsilon}(t) \rightarrow|t|$ as $t \rightarrow 0$ and $\left|g_{\epsilon}^{\prime}(t)\right| \leq 1$. Therefore, on using Fatou's Lemma and passing the limit $\epsilon \rightarrow 0$, we obtain

$$
\int_{Q} \frac{|| u(x)|-| u(y)||^{p(x, y)-2}(|u(x)|-|u(y)|)(\psi(x)-\psi(y))}{|x-y|^{N+s p(x, y)}} \mathrm{d} x \mathrm{~d} y \leq \int_{\Omega}\left(\left|\frac{\lambda}{|u|^{\gamma(x)-1} u}+\bar{f}(x, u)\right|\right) \psi \mathrm{d} x
$$

The inequality (4.7) holds true even for every $\psi \in X_{0}$. Now define $u_{k}=\min \left\{(u-1)^{+}, k\right\} \in X_{0}$ for each $k>0$. For any given $\beta>0$ and $\delta>0$ choose $\psi=\left(u_{k}+\delta\right)^{\beta}-\delta^{\beta}$ as the test function in (4.7). This gives

$$
\begin{aligned}
& \int_{Q} \frac{|| u(x)|-| u(y)||^{p(x, y)-2}(|u(x)|-|u(y)|)\left(\left(u_{k}(x)+\delta\right)^{\beta}-\left(u_{k}(y)+\delta\right)^{\beta}\right)}{|x-y|^{N+s p(x, y)}} \mathrm{d} x \mathrm{~d} y \\
& \quad \leq \int_{\Omega}\left|\frac{\lambda}{|u|^{\gamma(x)-1} u}+\bar{f}(x, u)\right|\left(\left(u_{k}+\delta\right)^{\beta}-\delta^{\beta}\right) \mathrm{d} x
\end{aligned}
$$

Now on applying Lemma 4.4 to the function $h(u)=\left(u_{k}+\delta\right)^{\beta}$ we obtain

$$
\begin{aligned}
& \int_{Q} \frac{\left|\left(\left(u_{k}(x)+\delta\right)^{\frac{\beta+p(x, y)-1}{p(x, y)}}-\left(u_{k}(y)+\delta\right)^{\frac{\beta+p(x, y)-1}{p(x, y)}}\right)\right|^{p(x, y)}}{|x-y|^{N+s p(x, y)}} \mathrm{d} x \mathrm{~d} y \\
& \leq \int_{Q}\left(\frac{(\beta+p(x, y)-1)^{p(x, y)}}{\beta p(x, y)^{p(x, y)}}\right) \frac{|| u(x)|-| u(y)||^{p(x, y)-2}(|u(x)|-|u(y)|)\left(\left(u_{k}(x)+\delta\right)^{\beta}-\left(u_{k}(y)+\delta\right)^{\beta}\right)}{|x-y|^{N+s p(x, y)}} \mathrm{d} x \mathrm{~d} y \\
& \leq\left(\frac{\left(\beta+p^{+}-1\right)^{p^{+}}}{\beta\left(p^{+}\right)^{p^{+}}}\right) \int_{Q} \frac{|| u(x)|-| u(y)||^{p(x, y)-2}(|u(x)|-|u(y)|)\left(\left(u_{k}(x)+\delta\right)^{\beta}-\left(u_{k}(y)+\delta\right)^{\beta}\right)}{|x-y|^{N+s p(x, y)}} \mathrm{d} x \mathrm{~d} y \\
& \leq\left(\frac{\left(\beta+p^{+}-1\right)^{p^{+}}}{\beta\left(p^{+}\right)^{p^{+}}}\right) \int_{\Omega}\left(\left|\frac{\lambda}{|u|^{\gamma(x)-1} u}\right|+|\bar{f}(x, u)|\right)\left(\left(u_{k}+\delta\right)^{\beta}-\delta^{\beta}\right) \mathrm{d} x \\
& \leq C_{1}\left(\frac{\left(\beta+p^{+}-1\right)^{p^{+}}}{\beta\left(p^{+}\right)^{p^{+}}}\right)\left[\int_{\Omega} \lambda|u|^{-\gamma(x)}\left(\left(u_{k}+\delta\right)^{\beta}-\delta^{\beta}\right)+\int_{\Omega}|u|^{\alpha(x)}\left(\left(u_{k}+\delta\right)^{\beta}-\delta^{\beta}\right) \mathrm{d} x\right] \\
& =C_{1}\left(\frac{\left(\beta+p^{+}-1\right)^{p^{+}}}{\beta\left(p^{+}\right)^{p^{+}}}\right)\left[\int_{\{u \geq 1\}} \lambda|u|^{-\gamma(x)}\left(\left(u_{k}+\delta\right)^{\beta}-\delta^{\beta}\right)+\int_{\{u \geq 1\}}|u|^{\alpha(x)}\left(\left(u_{k}+\delta\right)^{\beta}-\delta^{\beta}\right) \mathrm{d} x\right] \\
& \leq C C_{1}\left(\frac{\left(\beta+p^{+}-1\right)^{p^{+}}}{\beta\left(p^{+}\right)^{p^{+}}}\right)\left[\int_{\{u \geq 1\}}\left(1+|u|^{\alpha(x)}\right)\left(\left(u_{k}+\delta\right)^{\beta}-\delta^{\beta}\right) \mathrm{d} x\right] \\
& \leq C^{\prime}\left(\frac{\left(\beta+p^{+}-1\right)^{p^{+}}}{\beta\left(p^{+}\right)^{p^{+}}}\right)\left[\int_{\{u \geq 1\}}|u|^{\alpha(x)}\left(\left(u_{k}+\delta\right)^{\beta}-\delta^{\beta}\right) \mathrm{d} x\right] \\
& \leq C^{\prime}\left(\frac{\left(\beta+p^{+}-1\right)^{p^{+}}}{\beta\left(p^{+}\right)^{p^{+}}}\right)\left[\int_{\Omega}|u|^{\alpha^{+}}\left(\left(u_{k}+\delta\right)^{\beta}-\delta^{\beta}\right) \mathrm{d} x\right] \leq C^{\prime}\left(\frac{\left(\beta+p^{+}-1\right)^{p^{+}}}{\beta\left(p^{+}\right)^{p^{+}}}\right)\|u\|_{r^{*}}^{\alpha^{+}}\left\|\left(u_{k}+\delta\right)^{\beta}\right\|_{t},
\end{aligned}
$$

where $t=\frac{r^{*}}{r^{*}-\alpha^{+}},\left(t^{*}\right)<\left(p_{s}^{*}\right)^{-}$and $C=\max \{1,|\lambda|\}$. We now impose the Sobolev embedding 
theorem from [32, Theorem 1.1] to obtain

$$
\int_{Q} \frac{\left|\left(\left(u_{k}(x)+\delta\right)^{\frac{\beta+p(x, y)-1}{p(x, y)}}-\left(u_{k}(y)+\delta\right)^{\frac{\beta+p(x, y)-1}{p(x, y)}}\right)\right|^{p(x, y)}}{|x-y|^{N+s p(x, y)}} \mathrm{d} x \mathrm{~d} y \geq C\left\|\left(u_{k}+\delta\right)^{\frac{\beta+p(x, y)-1}{p(x, y)}}-\delta^{\frac{\beta+p(x, y)-1}{p(x, y)}}\right\|_{r^{*}}^{p(x, y)}
$$

where $C>0$. The triangle inequality and $\left(u_{k}+\delta\right)^{\beta+p(x, y)-1} \geq \delta^{p(x, y)-1}\left(u_{k}+\delta\right)^{\beta}$ implies

$$
\begin{array}{r}
{\left[\int_{\Omega}\left(\left(u_{k}+\delta\right)^{\frac{\beta+p(x, y)-1}{p(x, y)}}-\delta^{\frac{\beta+p(x, y)-1}{p(x, y)}}\right)^{r^{*}} \mathrm{~d} x\right]^{\frac{p(x, y)}{r^{*}} \geq\left(\frac{\delta}{2}\right)^{p(x, y)-1}}\left[\int_{\Omega}\left(u_{k}+\delta\right)^{\frac{r^{*} \beta}{p(x, y)}}\right]^{\frac{p(x, y)}{r^{*}}}} \\
-\delta^{\beta+p(x, y)-1|\Omega|} \frac{p(x, y)}{r^{*}} .
\end{array}
$$

Therefore, by using (4.10) in (4.9) and then from (4.8), we have

$\left\|\left(u_{k}+\delta\right)^{\frac{\beta}{p(x, y)}}\right\|_{r^{*}}^{p(x, y)} \leq C^{\prime}\left[C\left(\frac{2}{\delta}\right)^{p(x, y)-1}\left(\frac{\left(\beta+p^{+}-1\right)^{p^{+}}}{\beta\left(p^{+}\right)^{p^{+}}}\right)\|u\|_{r^{*}}^{\alpha^{+}}\left\|\left(u_{k}+\delta\right)^{\beta}\right\|_{t}+\delta^{\beta}|\Omega| \frac{p(x, y)}{r^{*}}\right]$.

Now by using Lemma 4.1, one can derive that

$$
\begin{aligned}
\delta^{\beta}|\Omega| \frac{p(x, y)}{r^{*}} & \leq\left(\frac{\left(\beta+p^{+}-1\right)^{p^{+}}}{\beta\left(p^{+}\right)^{p^{+}}}\right)|\Omega| \frac{p(x, y)}{r^{*}}-\frac{1}{t}\left\|\left(u_{k}+\delta\right)^{\beta}\right\|_{t} \\
& \leq\left(\frac{\left(\beta+p^{+}-1\right)^{p^{+}}}{\beta\left(p^{+}\right)^{p^{+}}}\right)|\Omega| \frac{p^{+}}{r^{*}}-\frac{1}{t}\left\|\left(u_{k}+\delta\right)^{\beta}\right\|_{t}
\end{aligned}
$$

Therefore, on using (4.12) in (4.11), we can deduce that

$$
\left\|\left(u_{k}+\delta\right)^{\frac{\beta}{p^{+}}}\right\|_{r^{*}}^{p^{+}} \leq C^{\prime}\left[\frac{1}{\beta}\left(\frac{\beta+p^{+}-1}{p^{+}}\right)^{p^{+}}\left\|\left(u_{k}+\delta\right)^{\beta}\right\|_{t}\left(\frac{C\|u\|_{r^{*}}^{\alpha^{+}}}{\delta^{p-1}}+|\Omega| \frac{p^{+}}{r^{*}}-\frac{1}{t}\right)\right] .
$$

Now choose, $\delta>0$ such that $\delta^{p-1}=C\|u\|_{r^{*}}^{\alpha^{+}}\left(|\Omega|^{\frac{p^{+}}{r^{*}}-\frac{1}{t}}\right)^{-1}$ and $\beta \geq 1$ with $\left(\frac{\beta+p^{+}-1}{p^{+}}\right)^{p^{+}} \leq \beta^{p^{+}}$. Further, by setting $\eta=\frac{r^{*}}{t p^{+}}>1$ (such a choice for $t$ and $r^{*}$ is possible) and $\tau=t \beta$ we can rewrite the inequality (4.13) as

$$
\left\|\left(u_{k}+\delta\right)\right\|_{\eta \tau} \leq\left(C|\Omega|^{\frac{p^{+}}{r^{*}}-\frac{1}{t}}\right)^{\frac{t}{\tau}}\left(\frac{\tau}{t}\right)^{\frac{t}{\tau}}\left\|\left(u_{k}+\delta\right)\right\|_{\tau}
$$


Set $\tau_{0}=t$ and $\tau_{m+1}=\eta \tau_{m}=\eta^{m+1} t$. Then after performing $m$ iterations, the inequality (4.14) reduces to

$$
\left\|\left(u_{k}+\delta\right)\right\|_{\tau_{m+1}} \leq\left(C|\Omega|^{\frac{p^{+}}{r^{*}}-\frac{1}{t}}\right)^{\left(\sum_{i=0}^{m} \frac{t}{\tau_{i}}\right)}\left(\prod_{i=0}^{m}\left(\frac{\tau_{i}}{t}\right)^{\frac{t}{\tau_{i}}}\right)^{p^{+}-1}\left\|\left(u_{k}+\delta\right)\right\|_{t}
$$

Again by using the fact $\eta>1$, we have

$$
\sum_{i=0}^{\infty} \frac{t}{\tau_{i}}=\sum_{i=0}^{\infty} \frac{1}{\eta^{i}}=\frac{\eta}{\eta-1}
$$

and

$$
\prod_{i=0}^{\infty}\left(\left(\frac{\tau_{i}}{t}\right)^{\frac{t}{\tau_{i}}}\right)^{p^{+}-1}=\eta^{\frac{\eta}{(\eta-1)^{2}}}
$$

Therefore, from (4.15), we get

$$
\left\|u_{k}\right\|_{\infty} \leq\left(C|\Omega|^{\frac{p^{+}}{r^{*}}-\frac{1}{t}}\right)^{\frac{\eta}{\eta-1}}\left(C^{\prime} \eta^{\frac{\eta}{(\eta-1)^{2}}}\right)^{p^{+}-1}\left\|\left(u_{k}+\delta\right)\right\|_{t}
$$

as $m \rightarrow \infty$ Furthermore, by applying the triangle inequality together with the fact $u_{k} \leq(u-1)^{+}$ in (4.16), we obtain

$$
\left\|u_{k}\right\|_{\infty} \leq C\left(\eta^{\frac{\eta}{(\eta-1)^{2}}}\right)^{p^{+}-1}\left(|\Omega|^{\frac{p^{+}}{r^{*}}-\frac{1}{t}}\right)^{\frac{\eta}{\eta-1}}\left(\left\|(u-1)^{+}\right\|_{t}+\delta|\Omega|^{\frac{1}{t}}\right)
$$

Finally letting $k \rightarrow \infty$ in (4.17), we get

$$
\left\|(u-1)^{+}\right\|_{\infty} \leq C\left(\eta^{\frac{\eta}{(\eta-1)^{2}}}\right)^{p^{+}-1}\left(|\Omega|^{\frac{p^{+}}{r^{*}}-\frac{1}{t}}\right)^{\frac{\eta}{\eta-1}}\left(\left\|(u-1)^{+}\right\|_{t}+\delta|\Omega|^{\frac{1}{t}}\right)
$$

Hence, we conclude that $u \in L^{\infty}(\bar{\Omega})$.

\section{Appendix}

We will begin with the following weak comparison principle.

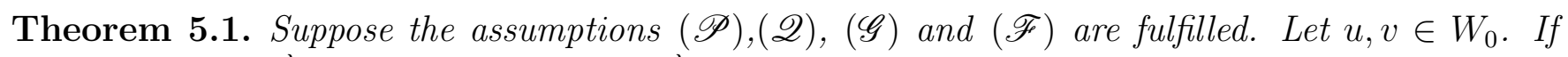
$\left(-\triangle_{p(x)}\right)^{s} u-\frac{\lambda}{|u|^{\gamma(x)-1} u} \leq\left(-\triangle_{p(x)}\right)^{s} v-\frac{\lambda}{|v|^{\gamma(x)-1} v}$ weakly in $\Omega$ and $u \leq v$ on $\Omega^{c}$, then $u \leq v$ in $\Omega$.

Proof. Since $\left(-\triangle_{p(x)}\right)^{s} u-\frac{\lambda}{|u|^{\gamma(x)-1} u} \leq\left(-\triangle_{p(x)}\right)^{s} v-\frac{\lambda}{|v|^{\gamma(x)-1} v}$ weakly in $\Omega$ and $u \leq v$ on $\Omega^{c}$, we have

$$
\left\langle\left(-\Delta_{p(x)}\right)^{s} v, \phi\right\rangle-\int_{\Omega} \frac{\lambda \phi}{|v|^{\gamma(x)-1} v} \mathrm{~d} x \geq\left\langle\left(-\Delta_{p(x)}\right)^{s} u, \phi\right\rangle-\int_{\Omega} \frac{\lambda \phi}{|u|^{\gamma(x)-1} u} \mathrm{~d} x, \forall \phi \geq 0 \in W_{0} .
$$

Now on choosing $\phi=(u-v)^{+}$as the test function in the inequality (5.1), it follows that

$$
\left\langle\left(-\Delta_{p(x)}\right)^{s} v-\left(-\Delta_{p(x)}\right)^{s} u,(u-v)^{+}\right\rangle-\int_{\Omega} \lambda(u-v)^{+}\left(\frac{1}{|v|^{\gamma(x)-1} v}-\frac{1}{|u|^{\gamma(x)-1} u}\right) \mathrm{d} x \geq 0 .
$$


Denote $Q(x, y)=\int_{0}^{1}|(u(x)-u(y))+t((v(x)-v(y))-(u(x)-u(y)))|^{p(x, y)-2} d t$. Then the identity

$$
|b|^{p(x, y)-2} b-|a|^{p(x, y)-2} a=(p(x, y)-1)(b-a) \int_{0}^{1}|a+t(b-a)|^{p(x, y)-2} \mathrm{~d} t
$$

with $a=v(x)-v(y), b=u(x)-u(y)$ gives

$$
\begin{aligned}
|u(x)-u(y)|^{p(x, y)-2}(u(x)-u(y)) & -|v(x)-v(y)|^{p(x, y)-2}(v(x)-v(y)) \\
& =(p(x, y)-1)\{(u(y)-v(y))-(u(x)-v(x))\} Q(x, y) .
\end{aligned}
$$

Observe that $Q(x, y)=Q(y, x) \geq 0$ for $(x, y) \in \mathbb{R}^{N} \times \mathbb{R}^{N}$. Furthermore, $Q(x, y)=0$ implies that $v(x)=v(y)$ and $u(x)=u(y)$. Set $\psi(x)=u(x)-v(x)$. Now choose $\phi=(u-v)^{+}$as the test function. Therefore, on using

$$
\psi=u-v=(u-v)^{+}-(u-v)^{-}
$$

we obtain

$$
[\psi(y)-\psi(x)][\phi(x)-\phi(y)]=-\left(\psi^{+}(x)-\psi^{+}(y)\right)^{2} \leq 0 .
$$

Now from the inequality (5.5), it follows that

$0 \geq\left\langle\left(-\Delta_{p(x, y)}\right)^{s} v-\left(-\Delta_{p(x, y)}\right)^{s} u,(v-u)^{+}\right\rangle=-\int_{\mathbb{R}^{N} \times \mathbb{N}^{N}} \frac{(p(x, y)-1) Q(x, y)\left(\psi^{+}(x)-\psi^{+}(y)\right)^{2}}{|x-y|^{N+s p(x, y)}} \mathrm{d} x \mathrm{~d} y \geq 0$.

This shows that the Lebesgue measure of $\Omega^{+}$, i.e., $\left|\Omega^{+}\right|=0$. In other words $v \geq u$ a.e. in $\Omega$.

The next Lemma is a generalization of the well known Lemma A.1 from [50].

Lemma 5.2. Let $\Omega$ be an open subset of $\mathbb{R}^{N}$ and $1<p^{-} \leq p(x) \leq p^{+}<\infty$. If $v_{n} \rightarrow u$ in $L^{p(x)}(\Omega)$, then there exists a subsequence $\left\{w_{n}\right\}$ of $\left\{v_{n}\right\}$ and a function $g \in L^{p(x)}(\Omega)$ such that $w_{n}(x) \rightarrow u(x)$ and $|u(x)|,\left|w_{n}(x)\right| \leq g(x)$ almost everywhere in $\Omega$.

Proof. Since $v_{n} \rightarrow u$ in $L^{p(x)}(\Omega)$ then we can assume, upto a subsequence that $v_{n}(x) \rightarrow u(x)$ almost everywhere in $\Omega$. Therefore there exists a subsequence $\left\{w_{n}\right\}$ of $\left\{v_{n}\right\}$ such that $\left\|w_{j+1}-w_{j}\right\|_{p(x)} \leq \frac{1}{2^{j}}$ for all $j \in \mathbb{N}$. Now define,

$$
g(x)=\left|w_{1}(x)\right|+\sum_{j=1}^{\infty}\left\|w_{j+1}(x)-w_{j}(x)\right\|_{p(x)}
$$

Thus $\left|w_{n}(x)\right| \leq g(x)$ a.e. in $\Omega$ and hence we get $|u(x)| \leq g(x)$.

\section{Acknowledgement}

The author S. Ghosh, thanks the Council of Scientific and Industrial Research (CSIR), India (09/983(0013)/2017-EMR-I) for the financial assistantship received to carry out this research work. D. Choudhuri thanks the Science and Engineering Research Board (SERB), India for the research grant (MTR/2018/000525) to carry out the research. R.Kr. Giri acknowledges the financial support and facilities received from the Mathematics Department, Technion - Israel Institute of Technology. 


\section{References}

\section{References}

[1] A. Ambrosetti, P. H. Rabinowitz, Dual variational methods in critical point theory and applications, Journal of Functional Analysis 14(4) (1973) 349-381. doi:10.1016/0022-1236(73)90051-7.

[2] D. C. Clark, A variant of the Lusternik-Schnirelman theory, Indiana University Mathematics Journal 22(1) (1972) 65-74. doi:10.1512/iumj.1972.22.22008.

[3] R. Kajikiya, A critical point theorem related to the symmetric mountain pass lemma and its applications to elliptic equations, Journal of Functional Analysis 225(2) (2005) 352-370. doi: $10.1016 / j \cdot j f a .2005 .04 .005$.

[4] F. Zhou, K. Wu, Infinitely many small solutions for a modified nonlinear Schrödinger equations, Journal of Mathematical Analysis and Applications 411(2) (2014) 953-959. doi:10.1016/j.jmaa.2013.09.058.

[5] G. Gu, W. Zhang, F. Zhao, Infinitely many positive solutions for a nonlocal problem, Applied Mathematics Letters 84 (2018) 49-55. doi:10.1016/j.aml.2018.04.010.

[6] G. Gu, W. Zhang, F. Zhao, Infinitely many sign-changing solutions for a nonlocal problem, Annali di Matematica Pura ed Applicata 197(5) (2018) 1429-1444. doi: $10.1007 /$ s10231-018-0731-2.

[7] A. C. Lazer, P. J. McKenna, On a singular nonlinear elliptic boundary value problem, Proceedings of the American Mathematical Society 111(3) (1991) 721-730. doi: $10.1090 /$ s0002-9939-1991-1037213-9.

[8] A. Canino, L. Montoro, B. Sciunzi, M. Squassina, Nonlocal problems with singular nonlinearity, Bulletin des Sciences Mathématiques 141(3) (2017) 223-250. doi:10.1016/j.bulsci.2017.01.002.

[9] L. Boccardo, L. Orsina, Semilinear elliptic equations with singular nonlinearities, Calculus of Variations and Partial Differential Equations 37(3-4) (2009) 363-380. doi: $10.1007 / \mathrm{s} 00526-009-0266-\mathrm{x}$.

[10] J. Giacomoni, I. Schindler, P. Takáč, Sobolev versus Hölder local minimizers and existence of multiple solutions for a singular quasilinear equation, Annali della Scuola Normale Superiore di Pisa-Classe di Scienze. Serie 5, 6(1) (2007) 117-158. URL: http://www. numdam.org/item/ASNSP_2007_5_6_1_117_0/.

[11] J. Giacomoni, K. Saoudi, Multiplicity of positive solutions for a singular and critical problem, Nonlinear Analysis: Theory, Methods \& Applications 71(9) (2009) 4060-4077. doi:10.1016/j.na.2009.02.087.

[12] K. Saoudi, S. Ghosh, D. Choudhuri, Multiplicity and hlder regularity of solutions for a nonlocal elliptic PDE involving singularity, Journal of Mathematical Physics 60(10) (2019) 101509. doi:10.1063/1.5107517. 
[13] T. Mukherjee, K. Sreenadh, On dirichlet problem for fractional p-laplacian with singular nonlinearity, Advances in Nonlinear Analysis 8(1) (2016) 52-72. doi:10.1515/anona-2016-0100.

[14] K. Saoudi, A critical fractional elliptic equation with singular nonlinearities, Fractional Calculus and Applied Analysis 20(6) (2017) 1507-1530. doi:10.1515/fca-2017-0079.

[15] R. Servadei, E. Valdinoci, Mountain pass solutions for non-local elliptic operators, Journal of Mathematical Analysis and Applications 389(2) (2012) 887-898. doi:10.1016/j.jmaa.2011.12.032.

[16] R. Servadei, E. Valdinoci, Variational methods for non-local operators of elliptic type, Discrete \& Continuous Dynamical Systems - A 33(5) (2013) 2105-2137. doi:10.3934/dcds.2013.33.2105.

[17] S. Ghosh, D. Choudhuri, Existence of infinitely many solutions for a nonlocal elliptic PDE involving singularity, Positivity 17(2) (2020) 97-115. doi:10.1007/s11117-019-00690-4.

[18] X. Fan, On the sub-supersolution method for $p(x)$-Laplacian equations, Journal of Mathematical Analysis and Applications 330(1) (2007) 665-682. doi:10.1016/j.jmaa.2006.07.093.

[19] J. Chabrowski, Y. Fu, Existence of solutions for $p(x)$-Laplacian problems on a bounded domain, Journal of Mathematical Analysis and Applications 306(2) (2005) 604-618. doi: $10.1016 /$ j.jmaa.2004.10.028.

[20] X.-L. Fan, Q.-H. Zhang, Existence of solutions for $p(x)$-Laplacian Dirichlet problem, Nonlinear Analysis. Theory, Methods \& Applications. An International Multidisciplinary Journal 52(8) (2003) 1843-1852. doi:10.1016/S0362-546X (02)00150-5.

[21] G. Bonanno, A. Chinnì, Existence results of infinitely many solutions for $p(x)$-Laplacian elliptic Dirichlet problems, Complex Variables and Elliptic Equations. An International Journal 57(11) (2012) 1233-1246. doi:10.1080/17476933.2012.662225.

[22] C. Ji, F. Fang, Infinitely many solutions for the $p(x)$-Laplacian equations without (AR)-type growth condition, Annales Polonici Mathematici 105(1) (2012) 87-99. doi:10.4064/ap105-1-8.

[23] V. D. Rădulescu, Nonlinear elliptic equations with variable exponent: old and new, Nonlinear Analysis. Theory, Methods \& Applications. An International Multidisciplinary Journal 121 (2015) 336-369. doi:10.1016/j.na.2014.11.007.

[24] Z. Tan, F. Fang, On superlinear $p(x)$-Laplacian problems without Ambrosetti and Rabinowitz condition, Nonlinear Analysis. Theory, Methods \& Applications. An International Multidisciplinary Journal 75(9) (2012) 3902-3915. doi:10.1016/j.na.2012.02.010.

[25] G. Bin, On superlinear $p(x)$-Laplacian-like problem without Ambrosetti and Rabinowitz condition, Bulletin of the Korean Mathematical Society 51(2) (2014) 409-421. doi:10.4134/BKMS.2014.51.2.409.

[26] A. Zang, $p(x)$-Laplacian equations satisfying Cerami condition, Journal of Mathematical Analysis and Applications 337(1) (2008) 547-555. doi:10.1016/j.jmaa.2007.04.007. 
[27] J. Gao, P. Zhao, Y. Zhang, Compact Sobolev embedding theorems involving symmetry and its application, NoDEA. Nonlinear Differential Equations and Applications 17(2) (2010) 161-180. doi:10.1007/s00030-009-0046-5.

[28] M. Mihăilescu, V. Rădulescu, On a nonhomogeneous quasilinear eigenvalue problem in Sobolev spaces with variable exponent, Proceedings of the American Mathematical Society 135(9) (2007) 2929-2937. doi:10.1090/S0002-9939-07-08815-6.

[29] N. T. Chung, Multiple solutions for a class of $p(x)$-Laplacian problems involving concave-convex nonlinearities, Electronic Journal of Qualitative Theory of Differential Equations (2013) No. 26, 17. doi:10.14232/ejqtde.2013.1.26.

[30] J. Yao, X. Wang, On an open problem involving the $p(x)$-Laplacian - a further study on the multiplicity of weak solutions to $p(x)$-Laplacian equations, Nonlinear Analysis. Theory, Methods \& Applications. An International Multidisciplinary Journal 69(4) (2008) 1445-1453. doi $10.1016 / \mathrm{j}$. na.2007.06.044.

[31] A. Bahrouni, Comparison and sub-supersolution principles for the fractional $p(x)$ Laplacian, Journal of Mathematical Analysis and Applications 458(2) (2018) 1363-1372. doi:10.1016/j.jmaa.2017.10.025.

[32] A. Bahrouni, , V. D. Rădulescu, On a new fractional Sobolev space and applications to nonlocal variational problems with variable exponent, Discrete \& Continuous Dynamical Systems 11(3) (2018) 379-389. doi:10.3934/dcdss.2018021.

[33] E. Azroul, A. Benkirane, M. Shimi, Eigenvalue problems involving the fractional $p(x)$-Laplacian operator, Advances in Operator Theory 4(2) (2019) 539-555. doi 10.15352/aot.1809-1420.

[34] N. T. Chung, H. Q. Toan, On a class of fractional Laplacian problems with variable exponents and indefinite weights, Collectanea Mathematica 71(2) (2020) 223-237. doi $10.1007 / \mathrm{s} 13348-019-00254-5$.

[35] J. I. Lee, J.-M. Kim, Y.-H. Kim, J. Lee, Multiplicity of weak solutions to non-local elliptic equations involving the fractional $p(x)$-Laplacian, Journal of Mathematical Physics 61(1) (2020) 011505, 13. doi:10.1063/1.5111786.

[36] C. F. Lorenzo, T. T. Hartley, Variable order and distributed order fractional operators, Nonlinear Dynamics 29(1-4) (2002) 57-98. doi:10.1023/a:1016586905654.

[37] Y. Chen, S. Levine, M. Rao, Variable exponent, linear growth functionals in image restoration, SIAM Journal on Applied Mathematics 66(4) (2006) 1383-1406. doi:10.1137/050624522.

[38] S. N. Antontsev, S. I. Shmarev, A model porous medium equation with variable exponent of nonlinearity: existence, uniqueness and localization properties of solutions, Nonlinear Analysis. Theory, Methods \& Applications. An International Multidisciplinary Journal 60(3) (2005) 515545. doi:10.1016/j.na.2004.09.026.

[39] V. V. Zhikov, Averaging of functionals of the calculus of variations and elasticity theory, Mathematics of the USSR-Izvestiya 29(1) (1987) 33-66. doi:10.1070/IM1987v029n01ABEH000958. 
[40] N. Laskin, Fractional quantum mechanics and Lévy path integrals, Physics Letters. A 268(4-6) (2000) 298-305. doi:10.1016/S0375-9601(00)00201-2.

[41] M. Kwaśnicki, Ten equivalent definitions of the fractional Laplace operator, Fractional Calculus and Applied Analysis 20(1) (2017) 7-51. doi 10.1515/fca-2017-0002.

[42] E. D. Nezza, G. Palatucci, E. Valdinoci, Hitchhiker's guide to the fractional sobolev spaces, Bulletin des Sciences Mathématiques 136(5) (2012) 521-573. doi:10.1016/j.bulsci.2011.12.004.

[43] V. D. Rădulescu, D. D. Repovšs, Partial Differential Equations with Variable Exponents: Variational Methods and Qualitative Analysis, first ed., Chapman and Hall/CRC, New York, 2015. doi:https://doi.org/10.1201/b18601.

[44] X. Fan, D. Zhao, On the spaces $L^{p(x)}(\Omega)$ and $W^{m, p(x)}(\Omega)$, Journal of Mathematical Analysis and Applications 263(2) (2001) 424-446. doi:10.1006/jmaa.2000.7617.

[45] O. Kováčik, J. Rákosník, On spaces $L^{p(x)}(\Omega)$ and $W^{k, p(x)}(\Omega)$, Czechoslovak Mathematical Journal 41(4) (1991) 592-618. URL: http://dml.cz/dmlcz/102493.

[46] D. E. Edmunds, J. Rákosník, Sobolev embeddings with variable exponent, Studia Mathematica 143(3) (2000) 267-293. doi $10.4064 / \mathrm{sm}-143-3-267-293$.

[47] D. E. Edmunds, J. Rákosník, Sobolev embeddings with variable exponent. II, Mathematische Nachrichten 246/247 (2002) 53-67. doi $10.1002 / 1522-2616(200212) 246: 1<53:$ : AID-MANA53>3.0.CD;2-T.

[48] U. Kaufmann, J. Rossi, R. Vidal, Fractional Sobolev spaces with variable exponents and fractional $p(x)$-Laplacians, Electronic Journal of Qualitative Theory of Differential Equations 2017(76) (2017) 1-10. doi:10.14232/ejqtde.2017.1.76.

[49] H. Brezis, E. Lieb, A relation between pointwise convergence of functions and convergence of functionals, Proceedings of the American Mathematical Society 88(3) (1983) 486-490. doi: $10.2307 / 2044999$.

[50] M. Willem, Minimax Theorems, volume 24 of Progress in Nonlinear Differential Equations and Their Applications, first ed., Birkhuser, Boston, 1997. doi:10.1007/978-1-4612-4146-1. 